\title{
Accretion-ejection morphology of the microquasar SS 433 resolved at sub-au scale ${ }^{\star}$
}

\author{
GRAVITY Collaboration $^{\star \star}$ : P.-O. Petrucci ${ }^{1}$, I. Waisberg ${ }^{2}$, J.-B. Le Bouquin ${ }^{1}$, J. Dexter ${ }^{2}$, G. Dubus ${ }^{1}$, K. Perraut ${ }^{1}$, \\ P. Kervella ${ }^{3,4}$, R. Abuter ${ }^{5}$, A. Amorim ${ }^{6}$, N. Anugu ${ }^{7}$, J.P. Berger ${ }^{5,1}$, N. Blind ${ }^{8}$, H. Bonnet ${ }^{5}$, W. Brandner ${ }^{9}$, A. Buron ${ }^{2}$, \\ É. Choquet ${ }^{3,10}$, Y. Clénet ${ }^{3}$, W. de Wit ${ }^{11}$, C. Deen ${ }^{2}$, A. Eckart ${ }^{12,13}$, F. Eisenhauer ${ }^{2}$, G. Finger ${ }^{5}$, P. Garcia ${ }^{7}$, \\ R. Garcia Lopez ${ }^{9}$, E. Gendron ${ }^{3}$, R. Genzel ${ }^{2}{ }^{14}$, S. Gillessen ${ }^{2}$, F. Gonte ${ }^{5}$, X. Haubois ${ }^{11}$, M. Haug ${ }^{2}$, , F. Haussmann ${ }^{2}$, \\ Th. Henning 9 , S. Hippler ${ }^{9}$, M. Horrobin ${ }^{12}$, Z. Hubert ${ }^{9}, 3$, L. Jochum ${ }^{5}$, L. Jocou ${ }^{1}$, Y. Kok ${ }^{2}$, J. Kolb ${ }^{11}$, M. Kulas ${ }^{9}$,

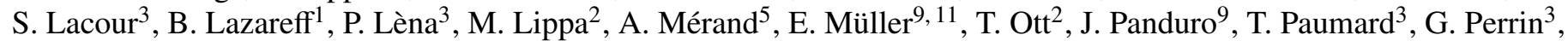 \\ O. Pfuhl ${ }^{2}$, J. Ramos ${ }^{9}$, C. Rau ${ }^{2}$, R.-R. Rohloff ${ }^{9}$, G. Rousset ${ }^{3}$, J. Sanchez-Bermudez ${ }^{9}$, S. Scheithauer ${ }^{9}$, M. Schöller ${ }^{5}$, \\ C. Straubmeier ${ }^{12}$, E. Sturm ${ }^{2}$, F. Vincent ${ }^{3}$, I. Wank ${ }^{12}$, E. Wieprecht ${ }^{2}$, M. Wiest ${ }^{12}$, E. Wiezorrek ${ }^{2}$, M. Wittkowski ${ }^{5}$, \\ J. Woillez ${ }^{5}$, S. Yazici ${ }^{2,12}$, and G. Zins ${ }^{11}$ \\ (Affiliations can be found after the references)
}

Received 25 April 2017 / Accepted 12 May 2017

\begin{abstract}
We present the first optical observation of the microquasar SS 433 at sub-milliarcsecond (mas) scale obtained with the GRAVITY instrument on the Very Large Telescope interferometer (VLTI). The 3.5-h exposure reveals a rich $K$-band spectrum dominated by hydrogen Br $\gamma$ and He I lines, as well as (red-shifted) emission lines coming from the jets. The $K$-band-continuum-emitting region is dominated by a marginally resolved point source $(<1$ mas) embedded inside a diffuse background accounting for $10 \%$ of the total flux. The jet line positions agree well with the ones expected from the jet kinematic model, an interpretation also supported by the consistent sign (i.e., negative/positive for the receding/approaching jet component) of the phase shifts observed in the lines. The significant visibility drop across the jet lines, together with the small and nearly identical phases for all baselines, point toward a jet that is offset by less than 0.5 mas from the continuum source and resolved in the direction of propagation, with a typical size of 2 mas. The jet position angle of $\sim 80^{\circ}$ is consistent with the expected one at the observation date. Jet emission so close to the central binary system would suggest that line locking, if relevant to explain the amplitude and stability of the $0.26 \mathrm{c}$ jet velocity, operates on elements heavier than hydrogen. The $\mathrm{Br} \gamma$ profile is broad and double peaked. It is better resolved than the continuum and the change of the phase signal sign across the line on all baselines suggests an East-West-oriented geometry similar to the jet direction and supporting a (polar) disk wind origin.
\end{abstract}

Key words. stars: individual: SS 433 - ISM: jets and outflows - techniques: interferometric - infrared: stars

\section{Introduction}

Discovered in the 1970s and initially catalogued as a star with an unusual emission-line spectrum (Clark \& Murdin 1978) SS 433 $(K=8.2)$ is a very well known microquasar. It is an eclipsing X-ray binary system, the primary component likely being a black hole of $\sim 5-16 M_{\odot}$ (e.g., Cherepashchuk et al. 2013 but see also Robinson et al. 2017) accreting matter from a massive companion star (e.g., Gies et al. 2002; Hillwig \& Gies 2008). Furthermore, it is located at $5.5 \pm 0.2 \mathrm{kpc}$ from the Sun (e.g., Blundell \& Bowler 2004) with an orbital period of 13.1 days (Fabrika 2004, hereafter F04) and is one of the most persistent sources of relativistic jets in our galaxy. Embedded in the radio nebula $\mathrm{W} 50$, it possesses a double-sided radio jet precessing on a period of 162.5 days and tracing out a cone of polar angle $\sim 20^{\circ}$ around a precession axis of position angle $\mathrm{PA} \sim 98^{\circ}$.

\footnotetext{
* Based on observations made with VLTI/Gravity instrument.

$\star \star$ GRAVITY is developed in a collaboration by the Max Planck Institute for extraterrestrial Physics, LESIA of Paris Observatory/CNRS/UPMC/Univ. Paris Diderot and IPAG of Université Grenoble Alpes/CNRS, the Max Planck Institute for Astronomy, the University of Cologne, the Centro Multidisciplinar de Astrofísica Lisbon and Porto, and the European Southern Observatory.
}

The jet of SS 433 has been intensively studied from arcminute- down to milliarcsecond (mas) scale in the radio. Very Large Baseline Interferometry observations show that jet signatures are already present at about 2 mas from the center (Paragi et al. 1999). SS 433 is the only microquasar whose jet is also observable in optical emission lines. The extremely large Doppler shifts seen in the optical lines are interpreted as the direct signature of jet material expelled at $\sim 0.26 c$ (Eikenberry et al. 2001). The powerful and continuous accretion flow is provided by the donor star at a rate of $\sim 10^{-4} M_{\odot} / \mathrm{yr}$, forming a complex structure around the system. Orbital phaseresolved optical spectra show evidence of an accretion disc in the inner region, which is also thought to power the ejection of matter (e.g., Perez \& Blundell 2009). The accretion disc is not directly observable due to the presence of a dense wind outflowing from the disc itself but was revealed by the detection of a pair of widely-separated, and hence rapidly rotating $\left(\sim 500 \mathrm{~km} \mathrm{~s}^{-1}\right)$, narrow components in the profile of the "stationary" (i.e., not associated with the jet) $\mathrm{Br} \gamma$ and $\mathrm{H} \alpha$ lines (Perez \& Blundell 2009; Perez M. \& Blundell 2010). The disc is expected to be perpendicular to the jet axis and precessing with it at the same period (e.g., Blundell et al. 2011). The IR spectrum of SS 433 is 


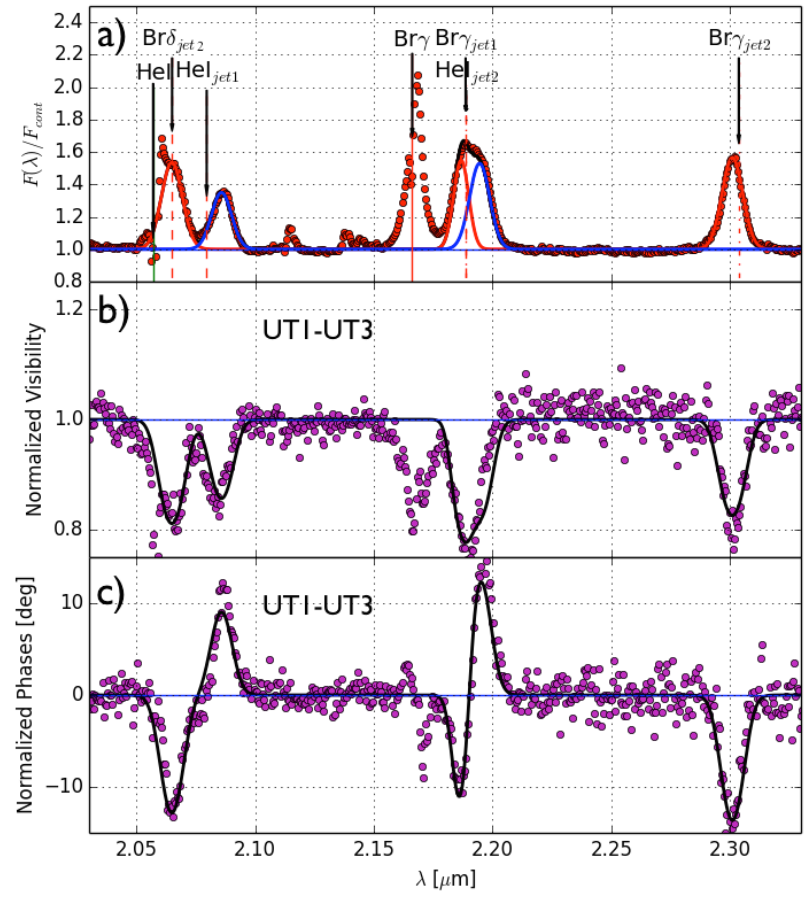

Fig. 1. a) Normalized $K$-band spectrum (with a binning over 2 spectral channels) obtained with GRAVITY. The $\mathrm{Br} \gamma$ and $\mathrm{He}$ I lines as well as the expected position of the corresponding jet lines (jet $1 /$ jet 2 for the approaching/receding jet) are indicated by vertical lines. We have also reported the expected position of the $\mathrm{Br} \delta_{\text {jet2 }}$ line from the receding jet. The solid blue/red line corresponds to the emission of the approaching/receding jet components. b) Visibility amplitudes and c) phases on the UT1-UT3 baseline (the visibility amplitudes and phases of all the baselines as well as the $u v$-plane at the time of the observation are shown in the Appendix, in Fig. B.1). For the phase, we follow the sign convention of Pauls et al. (2005), such that negative phases point to the baseline direction. The solid black line corresponds to the best fit model of the jet lines (see Sect. 3.2.2).

compatible with bremsstrahlung emission produced by the accretion disc wind, the estimated size at 2 microns of the accretion disc + wind system then being smaller than 1 mas (Fuchs et al. 2006).

We present in this letter the observation of SS 433 in the $K$ band performed in July 2016 with the GRAVITY instrument of the Very Large Telescope Interferometer (VLTI). The spectrointerferometric capabilities of GRAVITY allow us to resolve this microquasar at sub-mas spatial resolution for the first time in the optical. This gives us the opportunity to study on such scales and simultaneously study the properties of the different accretionejection components (jets, wind, disk), providing a new look at this famous source.

\section{Observations and data reduction}

We used GRAVITY (GRAVITY Collaboration et al. 2017; Eisenhauer et al. 2011) to perform spectral-differential interferometry on SS433 with the Unit Telescopes (UTs) of the VLTI. We recorded 18 data sets at high spectral resolution $(R=4000)$ over about $3.5 \mathrm{~h}$ on 2016, July 17 th. Each measurement consists of five exposures of $60 \mathrm{~s}$ integration time. We interlaced our observations with recordings on two interferometric calibrators,
HD 175322 and HD 181414 ${ }^{1}$. We used the GRAVITY standard pipeline (Lapeyrere et al. 2014) to reduce and calibrate the observations. We have checked that the different files are similar within errors. Then, to increase the signal-to-noise ratio, we merged all the files and binned the interferometric observables over two spectral channels. We used the interferometric data provided by the fringe tracker (working at a higher frequency, i.e., around $900 \mathrm{~Hz}$ ) to calibrate the continuum. The $u v$ plane at the date of observation is indicated in the Appendix (Fig. B.1).

\section{Results}

\subsection{K-band spectrum}

The $K$-band GRAVITY spectrum is plotted on the top panel of Fig. 1. It is very rich with hydrogen $\mathrm{Br} \gamma$ and $\mathrm{He} \mathrm{I}$ lines, as well as broad features around 2.08, 2.18, and $2.3 \mu \mathrm{m}$. The stationary $\mathrm{Br} \gamma$ line is clearly double peaked (see also Fig. 2) while the stationary He I line shows a P Cygni profile arising in the wind. We have overplotted in Fig. 1 the positions of the $\mathrm{He} \mathrm{I}, \mathrm{Br} \gamma$, and $\mathrm{Br} \delta$ approaching (hereafter jet1, pointing to East) and receding (hereafter jet2, pointing to West) jet lines as predicted by the standard kinematic model formula (see F04, and reference therein):

$1+z^{ \pm}=\gamma(1 \pm \beta \sin \theta \sin i \cos \psi \pm \beta \cos \theta \cos i)$,

where the - and + signs correspond to the jet 1 and jet 2 components, respectively, $\beta$ corresponds to the jet velocity in units of light speed, $\theta$ indicates the precession angle between the jets and the precessional axis, $i$ the angle between the precessional axis and the line of sight and $\psi$ the precessional phase at the observation date. We use the values determined by Eikenberry et al. (2001) that is, $\beta=0.2647, \theta=20.92^{\circ}$, and $i=78.05^{\circ}$. At the date of the GRAVITY observation, the estimated precessional phase was $\psi \sim 0.71$. The jet line positions computed with these parameter values (vertical dashed line in Fig. 1) agree relatively well with the position of the observed emission features, clearly supporting a jet origin. The fact that the signs of the phase shifts (Fig. 1c, see below) are the same for all the "jet1" lines and all the "jet2" lines, that is, positive (negative) for jet1 (jet2), also supports the idea that spatially resolved lines originate in the jets. These features are henceforth referred to here as jet lines. The parameters of our jet-lines fit (redshift, full width at half maximum (FWHM), equivalent width) are reported in Appendix (Table A.1).

We note that at the precession phase of $\sim 0.71$, the jet is almost in the plane of the sky (a favorable geometry to resolve the jet extension) and, due to the transverse Doppler effect, the receding and approaching shifts $z^{ \pm}$are both redshifts. Moreover, the approaching $\mathrm{He} \mathrm{I}$ and receding $\mathrm{Br} \gamma$ lines are very close and not distinguishable, producing a blended emission line profile around $2.18 \mu \mathrm{m}$. This precessional phase also corresponds to a near edge-on disk orientation, a precessional phase where PCygni profiles are commonly observed in $\mathrm{H}$ and $\mathrm{He} \mathrm{I}$ lines (F04).

\subsection{Visibilities and phases}

\subsubsection{Continuum}

The absolute visibility amplitudes of the continuum are all higher than 0.7 and display a systematic drop versus baseline

\footnotetext{
1 The "uniform disk" diameters in the $K$ band are $0.214 \pm 0.015$ mas and $0.143 \pm 0.010$ mas for HD 175322 (Sp. type F7V) and HD 181414 (Sp. type A2V) respectively.
} 
length. No closure phase is measurable in the continuum. A simple modeling with a Gaussian disk of the $K$-band-continuumemitting region shows that it is dominated by a marginally resolved source (typical size $\sim 0.8$ mas) embedded inside a diffuse background accounting for $10 \%$ of the total flux.

In the following, we normalize the visibility amplitudes and phases by their values in the continuum in order to work with differential quantities. Consequently the measured visibility amplitudes in the continuum (taken from the fringe tracker), hereafter abbreviated to $V_{\mathrm{c}}$ in equations, are included in the modeling. As an example, the normalized visibility amplitudes and phases for the UT1-UT3 baseline are shown in the middle and at the bottom of Fig. 1, respectively (the normalized visibility amplitudes and phases of the six baselines are plotted in the Appendix in Fig. B.1).

\subsubsection{Jet lines}

We decompose the jet lines contribution into two parts, $F_{\text {jet } 1}(\lambda) / F_{\text {jet} 2}(\lambda)$ from the jet 1 (approaching)/jet2 (receding) components separately as detailed in Appendix A. Looking at the visibility and phases, we infer that jet 1 and jet 2 are roughly symmetric around the continuum and that the $\mathrm{Br} \gamma$ and $\mathrm{He} \mathrm{I}$ lines are emitted from the same regions. Consequently the interferometric differential observables can be modeled with:

$V_{\mathrm{Norm}}(u, v, \lambda)=\frac{F_{\mathrm{jet} 1}(\lambda) V(u, v)+F_{\mathrm{jet} 2}(\lambda) V(-u,-v)+V_{c}(u, v)}{V_{c}(u, v)\left[F_{\mathrm{jet} 2}(\lambda)+F_{\mathrm{jet} 1}(\lambda)+1\right]}$,

where $V(u, v)$ is the Fourier Transform of the geometrical model for Jet1.

The significant visibility drop across the lines (with deeper drops on the longest baselines), together with the significant $\left(>10^{\circ}\right.$ for each spectral line) and nearly identical phases for all baselines, point toward a significantly resolved overall jet geometry, with a typical size of 2 mas and only slightly offset from the continuum, typically by less than 1 mas.

This is confirmed by tentative adjustments with components located further away (one blob, several blobs, varying PA, flux ratio, elongation, and intensity profile, etc.). Secondary minima exist in the $\chi_{r}^{2}$ cube for solutions located further away (typically 10 mas with $\mathrm{PA} \approx 70 \mathrm{deg}$ and 15 mas with $\mathrm{PA} \approx 90 \mathrm{deg}$ ). However the fit qualities are low $\left(\chi_{r}^{2}>3\right)$ and they predict an incorrect sign of the visibility phase for at least one baseline. In fact, fitting all jet lines together under the assumption that they have similar origins and structures makes the fit rather well constrained because of the additional spatial frequency coverage. We conclude that elongated models $(>2.5$ mas) for the jet are disfavored with the present dataset.

A model composed of a single resolved and slightly offset Gaussian provides a statistically better fit $\left(\chi_{r}^{2}=1.36\right)$. An elongated (along the jet axis) Gaussian blob gives a statistically similar fit $\left(\chi^{2}=1.34\right)$. We decided to explore a more abrupt intensity profile for the jet:

$V(u, v, s, a)=\frac{\exp (2 i \pi f a)}{1+2 i \pi f s}$,

with $f=v \cos (\mathrm{PA})-u \sin (\mathrm{PA})$. This is the Fourier Transform of an exponentially decreasing profile $\exp (-(r-a) / s) H(r-a)$, where $H(r)$ is the Heaviside function. It is limited to the positive ordinates, decays on a spatial scale, $s$, and is translated by $a$ from the continuum position. Positive $r$ are defined toward PA (North

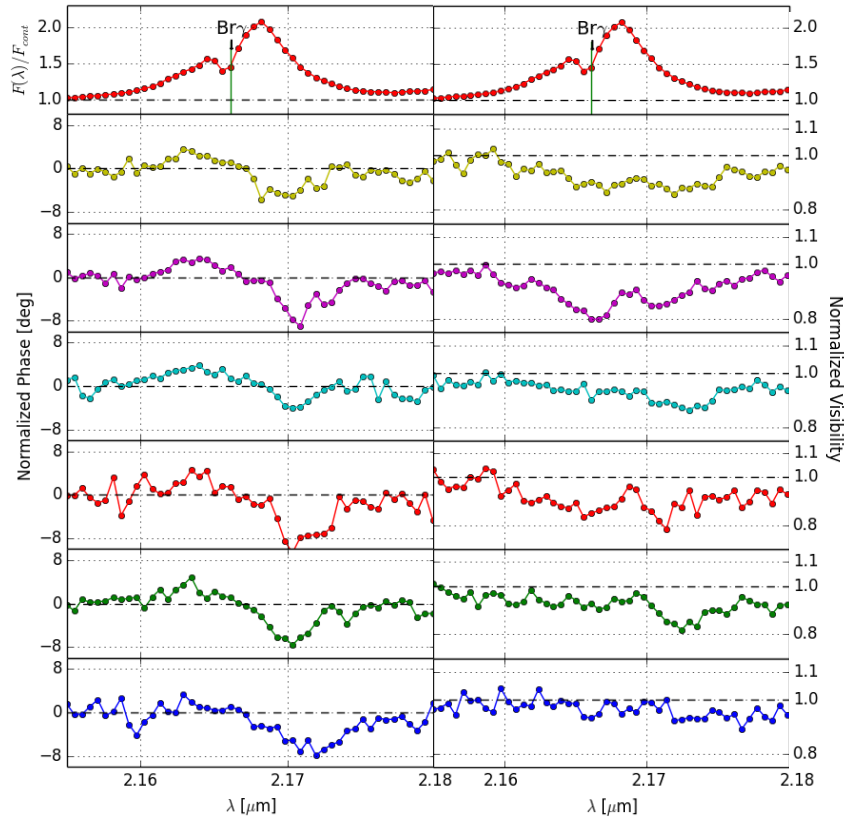

Fig. 2. Normalized spectrum (top), visibility phases (left) and amplitudes (right) around the $\mathrm{Br} \gamma$ line with a spectral binning of 2 . The symbol colors correspond to the baseline colors indicated in Fig. B.1.

to East), and the model is infinitely thin in the direction perpendicular to PA. The fit is significantly better $\left(\chi_{r}^{2}=0.89\right)$. The best fit parameter values (with their $3 \sigma$ errors $^{2}$ ) are $s=1.7 \pm 0.6$ mas, $a=-0.15 \pm 0.34$ mas, and PA $=75 \pm 20^{\circ}$. The resulting best fit is reported in Fig. 1 represented by a black solid line. We tested the thickness of the jet in the transverse direction by convolving the intensity profile with a Gaussian. The jet profile is unresolved in the transverse direction, with an upper limit of its transverse size of $\sim 1.2$ mas at $3 \sigma$. This upper limit is relatively large, however, due to the coincidental alignment of the baselines with the jet PA (see the $u v$ plane in the Appendix in Fig. B.1).

\subsubsection{Stationary lines: $\mathrm{Br} \gamma$}

The Br $\gamma$ profile is broad and double peaked. The visibilities clearly drop across the line for all the baselines, with a decrease that can reach $20 \%$ with respect to the continuum (Fig. 2). The drop is also deeper for longer baselines. The emitting region size is found to be $\sim 1$ mas, similar to the extension of the jets and a little larger than the continuum. The phases in the red part are significantly larger (in absolute value) than in the blue part. They show a similar behavior for all the baselines, starting from positive values of a few degrees in the blue part of the line, and going to negative values in the red part (Fig. 2). This suggests an East-West-oriented geometry, that is, in a direction similar to the jet one. This East-West direction disfavors an origin from the accretion disk, which is expected to be perpendicular to the jet axis (i.e., South-North). A (rather polar) disk wind is a more natural candidate. The change of the phase sign across the line can be explained then by the presence of an approaching (positive phase) and a receding (negative phase) component in the wind.

2 To compute the errors, we assume 60 observables ( 6 baselines $\times$ 3 lines $(\mathrm{Br} \gamma, \mathrm{Br} \delta$ and $\mathrm{He} \mathrm{I}) \times 2$ jet components (only 1 for $\mathrm{Br} \delta) \times 2$ interferometric measurements (visibility amplitude and phase)) and 3 model parameters ( $a, s$ and PA), giving 57 degrees of freedom. 


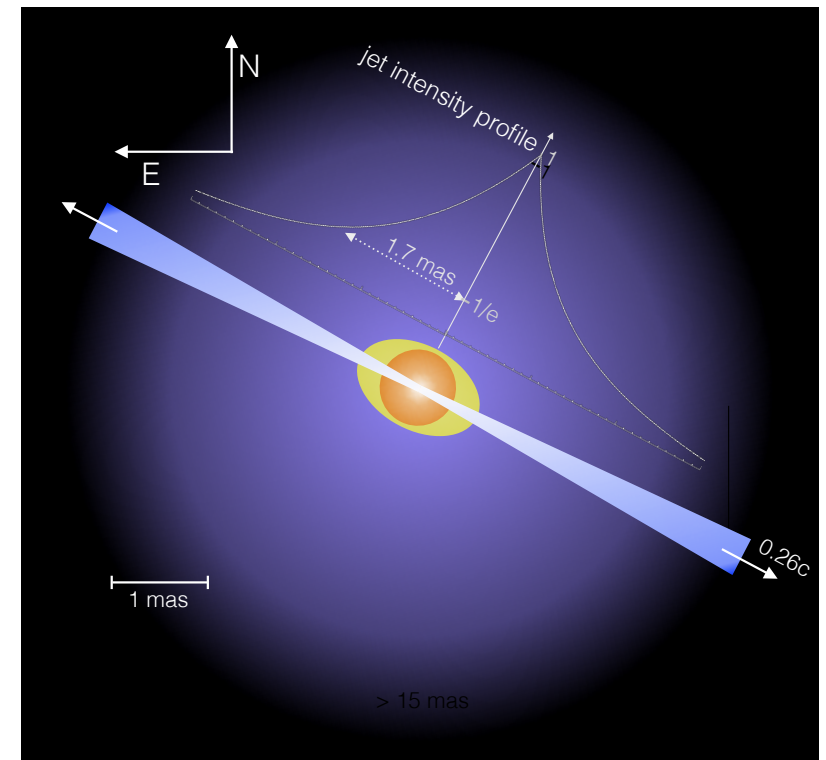

Fig. 3. Sketch of the inner region of SS 433. Most (90\%) of the infrared continuum comes from a partially resolved central source of typical size $\sim 0.8$ mas (central red area). The $10 \%$ continuum flux left is produced by a completely resolved background on a larger scale ( $>15$ mas, background area). The $\mathrm{Br} \gamma$-line-emitting region size is found to be $\sim 1$ mas and is dominated by an East-West component, in the direction of the jet (yellow area). The measured position angle of the jet is $\sim 80^{\circ}$. The jet profile is unresolved in the transverse direction $(<1.2$ mas). The intensity profile along the jet axis is best characterized by an emission peaking at the continuum position and decreasing exponentially on a scale of 1.7 mas.

\section{Discussion}

Our GRAVITY observations of SS 433 allow us to resolve its accretion-ejection structure at sub-mas scale. A sketch summarizing our results is shown in Fig. 3. Most (90\%) of the infrared continuum comes from a partially resolved central source of typical size $\sim 0.8$ mas potentially dominated by emission from outflowing material forming a circumbinary disc (Fuchs et al 2006). The $10 \%$ continuum flux left over could be produced by a completely resolved background on a larger scale (>15 mas). This background shows no asymmetry (no closure phase is measurable in the continuum) that could suggest a link with the jets. On the other hand it is relatively extended. It could be the circumbinary material excreted by the binary system and illuminated by the inner region emission (Fabrika 1993). Jet lines are clearly present in the spectrum. The measured position angle of the jet is fully consistent with the PA $\left(\sim 80^{\circ}\right)$ derived from the kinematic model at a precession phase $\psi=0.71$. The jet profile is unresolved in the transverse direction $(<1.2$ mas at $3 \sigma)$, as expected since both optical and X-ray emission line widths indicate a jet opening angle $\theta_{j} \approx 1.5^{\circ}$ (Borisov \& Fabrika 1987; Marshall et al. 2002). The intensity profile along the jet axis is best characterized in our data by an emission peaking at the continuum position and decreasing exponentially on a scale of 1.7 mas $=1.4 \times 10^{14} \mathrm{~cm}$.

This profile for the jet intensity is certainly not unique. However, the relatively strong decrease of the visibility amplitude across the jet lines for all the baselines, as well as the similar phase behavior of the jet lines produced by the same jet component, strongly support a sharply decreasing two-sided intensity profile for the jet.
During the $\sim 4 \mathrm{~h}$ observation, the jet material traveling at $0.26 \mathrm{c}$ moves by $\sim 1.3$ mas, that is, of the order of the size of the jet structure. This implies that rapid flux decay must occur during propagation along the jet to explain the exponential decay of the jet intensity profile. Interestingly, an exponential profile was also used by Borisov \& Fabrika (1987) to fit the flux decay timescale of individual kinematic components in the $\mathrm{H} \alpha$ profile. They found an offset of the jet emission by $4 \times 10^{14} \mathrm{~cm}(4.8 \mathrm{mas})$ from the core and with a larger spatial extent of $6.7 \times 10^{14} \mathrm{~cm}$ (8.1 mas) (see also F04). A direct comparison is difficult however given the non-simultaneity of these observations and the known variability of the jet structure. Moreover, the NIR and optical lines may have different emissivity profiles (e.g., because of increased extinction in the optical compared to the NIR).

Our observation unambiguously fixes the size of the jet infrared line emission region $\left(\sim 1.4 \times 10^{14} \mathrm{~cm}\right)$. Given the average jet-line luminosities, this size is far too large for the (conical) jet to be entirely filled with emitting gas, and a relatively small clump filling factor $\left(\sim 10^{-5}\right)$ is required (e.g., Davidson \& McCray 1980; Begelman et al. 1980; Panferov \& Fabrika 1997). Clumping is likely to be due to thermal instabilities in a hot outflow, with the outflow initially driven by radiation pressure in the funnel of an accretion disk (Davidson \& McCray 1980). X-ray line variability limits the hot outflow region to $<4 \times 10^{13} \mathrm{~cm}$ (Marshall et al. 2013). Our observation shows that the IR emission is displaced by less than 0.2 mas $=1.6 \times 10^{13} \mathrm{~cm}$ from the central binary system, confirming that clumping originates early on in the hot outflow. It also suggests that line locking (Milgrom 1979), if relevant to explain the amplitude and stability of the $0.26 c$ jet velocity, operates on elements heavier than hydrogen to be efficient on this small scale (Shapiro et al. 1986). Models show that continuous heating of the clumps is required to explain optical emission on a scale of $10^{14} \mathrm{~cm}$ (Brinkmann et al. 1988). Future comparison of observed and theoretical intensity profiles may shed light on this heating process (Brinkmann \& Kawai 2000).

The stationary $\operatorname{Br} \gamma$ line shows a broad and double peaked profile and the interferometric observables suggest a geometry dominated by an East-West component, in the direction of the jet, such as a (rather polar) disk wind. Then, both receding and approaching components must be present to explain the change of sign of the phases across the line. Taking the wavelength of the phase extrema as the corresponding blue and redshifted line produced by the wind, we infer a velocity of $\sim 600 \mathrm{~km} \mathrm{~s}^{-1}$ and $\sim 2000 \mathrm{~km} \mathrm{~s}^{-1}$ for the approaching and receding flow, respectively. We also observe a similar dissymmetry in the phases, which are significantly smaller in the blue part of the line. Absorption effects could play a role here. Indeed at the precessional phase of the GRAVITY observation, the accretion disk has a near edge-on orientation and strong absorption from the wind could affect the line profile (like the P Cygni profile of He I), especially in its blue part.

The results presented here demonstrate the potential of spectro-interferometry to dissect the super-Eddington outflows and jets of SS 433. Additional insights will be gained in the future by monitoring this source at different precession and orbital phases with GRAVITY to obtain spectro-interferometric constraints on the stationary and jet-line variability.

Acknowledgements. This work is based on observations made with ESO Telescopes at the La Silla Paranal Observatory, programme ID 60.A-9102. It has been supported by a grant from LabEx OSUG@2020 (Investissements d'avenir - ANR10LABX5) and in part by the National Science Foundation under Grant No. NSF PHY-1125915. It has made use of the Jean-Marie Mariotti Center 
Aspro2 and SearchCal services ${ }^{3}$. P.O.P. and G.D. acknowledge financial support from CNES and the French PNHE. J.D. was supported by a Sofja Kovalevskaja Award from the Humboldt Foundation of Germany. E.C. is supported by NASA through a Hubble Fellowship grant HST-HF2-51355 awarded by STScI, operated by AURA, Inc. We thank the ESO/VLTI team for their constant support. We also thank the technical, administrative, and scientific staff of the participating institutes and the observatory for their extraordinary support during the development, installation, and commissioning of GRAVITY.

\section{References}

Begelman, M., Hatchett, S., McKee, C., et al. 1980, ApJ, 238, 722 Blundell, K. M., \& Bowler, M. G. 2004, ApJ, 616, L159

Blundell, K. M., Schmidtobreick, L., \& Trushkin, S. 2011, MNRAS, 417, 2401

Borisov, N. V., \& Fabrika, S. N. 1987, Sov. Astron. Lett., 13, 200

Brinkmann, W., \& Kawai, N. 2000, A\&A, 363, 640

Brinkmann, W., Fink, H., Massaglia, S., et al. 1988, A\&A, 196, 313

Cherepashchuk, A., Sunyaev, R., Molkov, S., et al. 2013, MNRAS, 436, 2004

Clark, D. H., \& Murdin, P. 1978, Nature, 276, 44

Davidson, K., \& McCray, R. 1980, ApJ, 241, 1082

Eikenberry, S. S., Cameron, P. B., Fierce, B. W., et al. 2001, ApJ, 561, 1027

Eisenhauer, F., Perrin, G., Brandner, W., et al. 2011, The Messenger, 143, 16

Fabrika, S. N. 1993, MNRAS, 261, 241

Fabrika, S. 2004, Astrophys. Space Phys. Rev., 12, 1

Fuchs, Y., Koch Miramond, L., \& Ábrahám, P. 2006, A\&A, 445, 1041

Gies, D. R., Huang, W., \& McSwain, M. V. 2002, ApJ, 578, L67

GRAVITY Collaboration, Abuter, R., Accardo, M., et al. 2017, A\&A, in press DOI: $10.1051 / 0004-6361 / 201730838$

Hillwig, T. C., \& Gies, D. R. 2008, ApJ, 676, L37

Lapeyrere, V., Kervella, P., Lacour, S., et al. 2014, in Optical and Infrared Interferometry IV, Proc. SPIE, 9146, 91462D

Marshall, H. L., Canizares, C. R., \& Schulz, N. S. 2002, ApJ, 564, 941

Marshall, H. L., Canizares, C. R., Hillwig, T., et al. 2013, ApJ, 775, 75

Milgrom, M. 1979, A\&A, 78, L9

Panferov, A. A., \& Fabrika, S. N. 1997, Astron. Rep., 41, 506

Paragi, Z., Vermeulen, R. C., Fejes, I., et al. 1999, A\&A, 348, 910

Pauls, T., Young, J., Cotton, W., \& Monnier, J. 2005, PASP, 117, 1255

Perez, M. S., \& Blundell, K. M. 2009, MNRAS, 397, 849
Perez M., S., \& Blundell, K. M. 2010, MNRAS, 408, 2

Robinson, E. L., Froning, C. S., Jaffe, D. T., et al. 2017, ApJ, in press [arXiv: 1704.08166]

Shapiro, P. R., Milgrom, M., \& Rees, M. J. 1986, ApJS, 60, 393

1 Univ. Grenoble Alpes, CNRS, IPAG, 38000 Grenoble, France e-mail: pierre-olivier . petrucci@univ-grenoble-alpes.fr

2 Max Planck Institute for extraterrestrial Physics, Giessenbachstr., 85748 Garching, Germany

3 LESIA, Observatoire de Paris, PSL Research University, CNRS, Sorbonne Universités, UPMC Univ. Paris 06, Univ. Paris Diderot, Sorbonne Paris Cité, 75005 Paris, France

4 Unidad Mixta Internacional Franco-Chilena de Astronomía (CNRS UMI 3386), Departamento de Astronomía, Universidad de Chile, Camino El Observatorio 1515, Las Condes, Santiago, Chile

5 European Southern Observatory, Karl-Schwarzschild-Str. 2, 85748 Garching, Germany

6 CENTRA and Universidade de Lisboa - Faculdade de Ciências, Campo Grande, 1749-016 Lisboa, Portugal

7 CENTRA and Universidade do Porto - Faculdade de Engenharia, 4200-465 Porto, Portugal

8 Observatoire de Genève, Université de Genève, 51 Ch. des Maillettes, 1290 Versoix, Switzerland

9 Max-Planck-Institut für Astronomie, Königstuhl 17, 69117 Heidelberg, Germany

10 Hubble Fellow, Jet Propulsion Laboratory, California Institute of Technology, 4800 Oak Grove Drive, Pasadena, CA 91109, USA

11 European Southern Observatory, Casilla 19001, Santiago 19, Chile

12 1. Physikalisches Institut, Universität zu Köln, Zülpicher Str. 77, 50937 Köln, Germany

13 Max-Planck-Institute for Radio Astronomy, Auf dem Hügel 69, 53121 Bonn, Germany

14 Department of Physics, Le Conte Hall, University of California, Berkeley, CA 94720, USA

\footnotetext{
3 Available at http: //www . jmmc . fr
} 


\section{Appendix A: Jet lines spectral and interferometric signature modeling}

We decompose the contribution from the jet1 (approaching) and jet2 (receding) components separately as follows:

$F_{\text {Norm }}(\lambda)=F_{\text {jet1 }}(\lambda)+F_{\text {jet2 }}(\lambda)+1$,

$F_{\text {jet1 }}(\lambda)=\operatorname{He~}_{\text {jet } 1}+\operatorname{Br} \gamma_{\text {jet } 1}$,

$F_{\text {jet2 }}(\lambda)=\mathrm{He}_{\text {jet } 2}+\operatorname{Br} \gamma_{\text {jet } 2}+\operatorname{Br} \delta_{\text {jet } 2}$,

where $\mathrm{He} \mathrm{I}_{\text {jet } 1 / \mathrm{jet} 2}, \mathrm{Br} \gamma_{\text {jet } 1 / \text { jet } 2}$, and $\mathrm{Br} \delta_{\text {jet } 2}$ are the flux ratios (fixed using the average spectrum) between the lines and the continuum. Assuming that the $\mathrm{Br} \gamma, \mathrm{Br} \delta$, and $\mathrm{He}$ I lines are emitted from the same regions, the interferometric differential observables are given then by Eq. (2). The characteristics of the different jet lines (redshift, FWHM, EW) are reported in Table A.1.
Table A.1. Properties of each jet line.

\begin{tabular}{lcccc}
\hline \hline Name & $\begin{array}{c}\text { Rest wavelength } \\
(\mu \mathrm{m})\end{array}$ & Redshift & $\begin{array}{c}F W H M \\
\left(\mathrm{~km} \mathrm{~s}^{-1}\right)\end{array}$ & $\begin{array}{c}E W \\
(\AA)\end{array}$ \\
\hline $\mathrm{Br} \gamma_{\text {jet1 }}$ & 2.166 & 0.0132 & 1364 & 56 \\
$\mathrm{Br} \gamma_{\text {jet2 }}$ & 2.166 & 0.0624 & 1347 & 62 \\
$\mathrm{Br} \delta_{\text {jet2 }}$ & 1.944 & 0.0622 & 1510 & 59 \\
$\mathrm{He} \mathrm{I}_{\text {jet1 }}$ & 2.057 & 0.0140 & 1429 & 37 \\
$\mathrm{He} \mathrm{I}_{\text {jet2 }}$ & 2.057 & 0.0631 & 1027 & 44 \\
\hline
\end{tabular}

\section{Appendix B: Normalized visibility amplitudes and phases of the six baselines}

The $u v$ plane at the time of the observation, the $K$-band GRAVITY spectrum as well as the visibility amplitudes and phases for the six baselines are plotted in Fig. B.1.
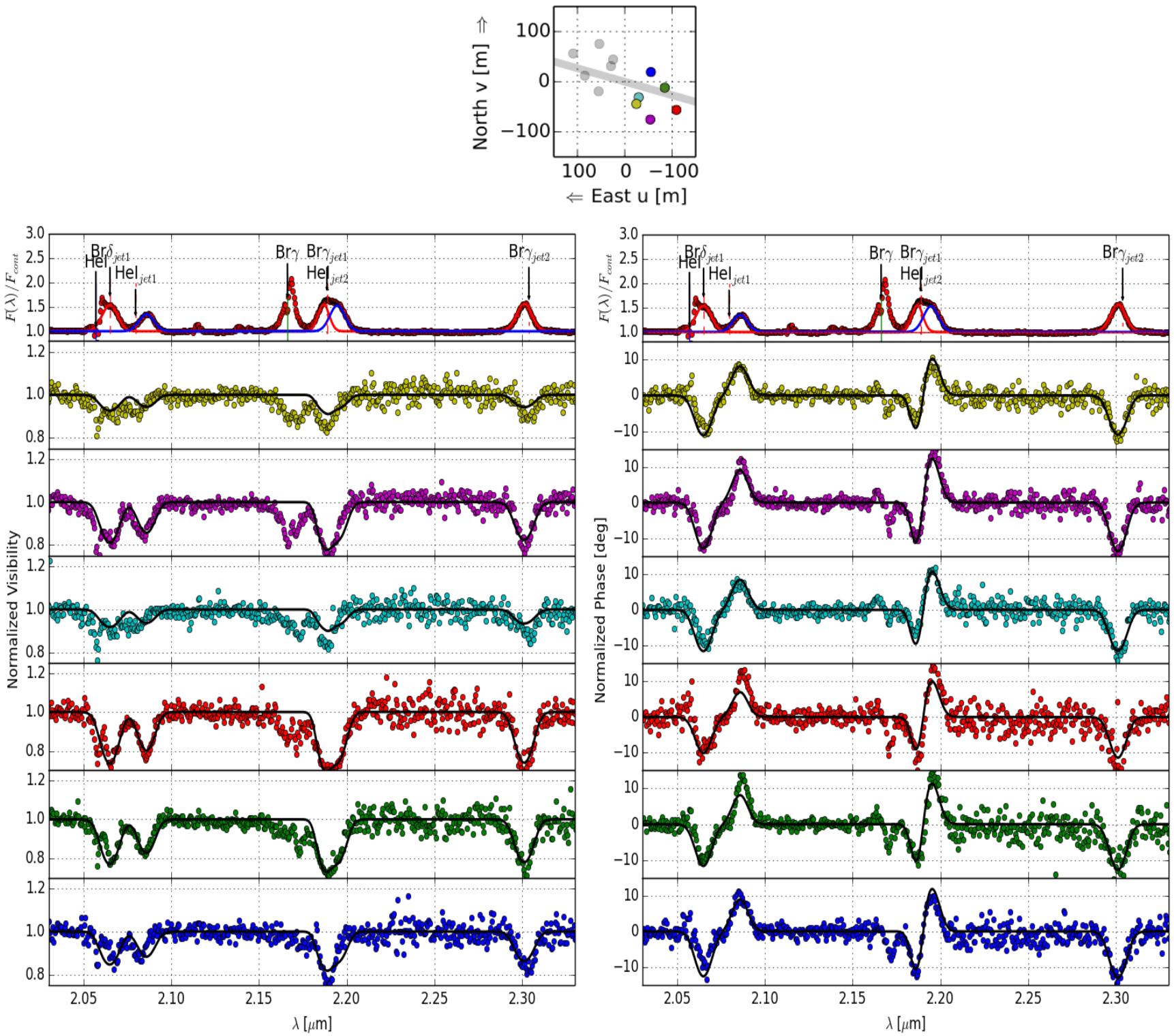

Fig. B.1. (Top) $u v$ plane at the time of the observation (average over the full exposure) with the different baselines indicated by colored points. The gray line represents the expected jet PA. (Bottom) $K$-band GRAVITY spectrum (the solid blue/red line corresponds to the emission of the approaching/receding jet components) as well as the visibility amplitudes (left) and phases (right) for the six baselines. The solid line corresponds to the best fit model of the jet lines (see Sect. 3.2.2). The symbol colors correspond to the baseline colors indicated in the $u v$ plane. 